\title{
Oxidase mimetic activity of metalloporphyrin-containing porous organic polymer and its applications for colorimetric detection of both ascorbic acid and glutathione
}

Ding Guo ${ }^{1}$, Cheng $\mathrm{Li}^{1}$, Genyan Liu ${ }^{1}$, Xiaogang Luo ${ }^{1,2}$, Fengshou $\mathrm{Wu}^{1, *}$

${ }^{1}$ Hubei key Laboratory of Novel Reactor and Green Chemical Technology, Key Laboratory for Green Chemical Process of Ministry of Education, School of Chemical Engineering and Pharmacy, Wuhan Institute of Technology, Wuhan, 430205, P. R. China.

${ }^{2}$ School of Materials Science and Engineering, Zhengzhou University, Zhengzhou, 450001, P. R. China.

Number of Pages: 5

Number of Figures: 9

\section{Experiment sections}

\section{Materials and measurements}

Pyrrole, benzaldehyde, cobalt acetate tetrahydrate, dimethoxymethane and other reagents used to synthesize Co-POP were obtained from Aladdin. TMB, AA, GSH and other reagents used for analysis were purchased from Inno-chem. The fresh fruits were purchased from shops and the human serum was supplied by the affiliated hospital of Wuhan Institute of Technology. Scanning electron microscope (SEM) images were obtained using a JEOL630-F field-emission scanning electron microscopy. Transmission electron microscope (TEM) images were collected with a JEM2100 TEM instrument. Powder X-ray diffraction (XRD) patterns were acquired at room temperature on a D8 Advance X-ray diffractometer (Bruker AXS Germany) with $\mathrm{Cu} \mathrm{Kr}$ radiation, and the recording speed was $1^{\circ} \mathrm{min}^{-1}$. X-ray photoelectron spectra (XPS) was performed on an ESCALAB multifunctional imaging electron spectrometer (Thermo). The Fourier transform infrared (FT-IR) spectra were measured at wavenumbers of $400-4000 \mathrm{~cm}^{-1}$ using a NICOLET6700 spectrometer (ABB Bomen Canada) with $\mathrm{KBr}$ pellets. Absorption spectra were obtained with a UV-vis spectrophotometer (Shimadzu). 


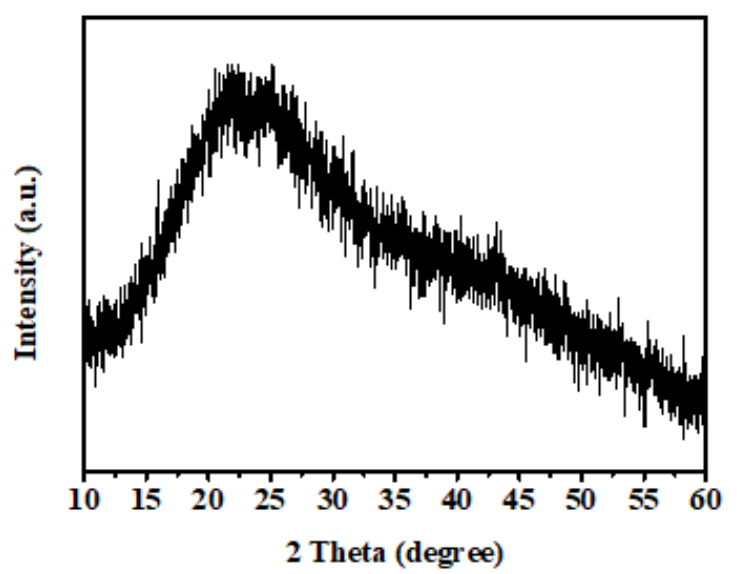

Figure S1. The PXPD of Co-POP.

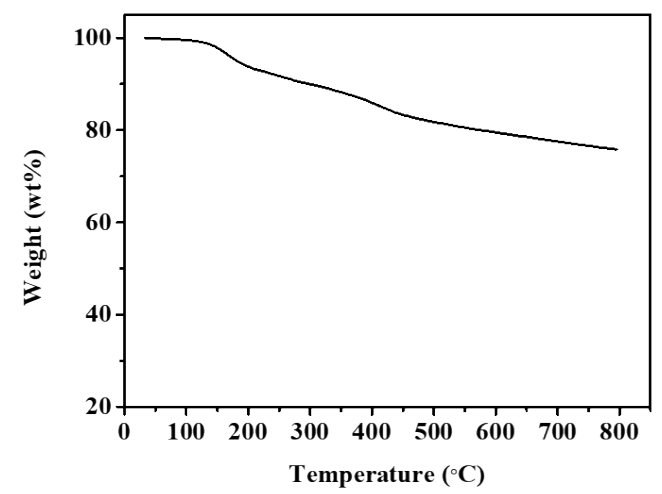

Figure S2. The TGA of Co-POP with a heating rate of $10{ }^{\circ} \mathrm{C} \mathrm{min}^{-1}$
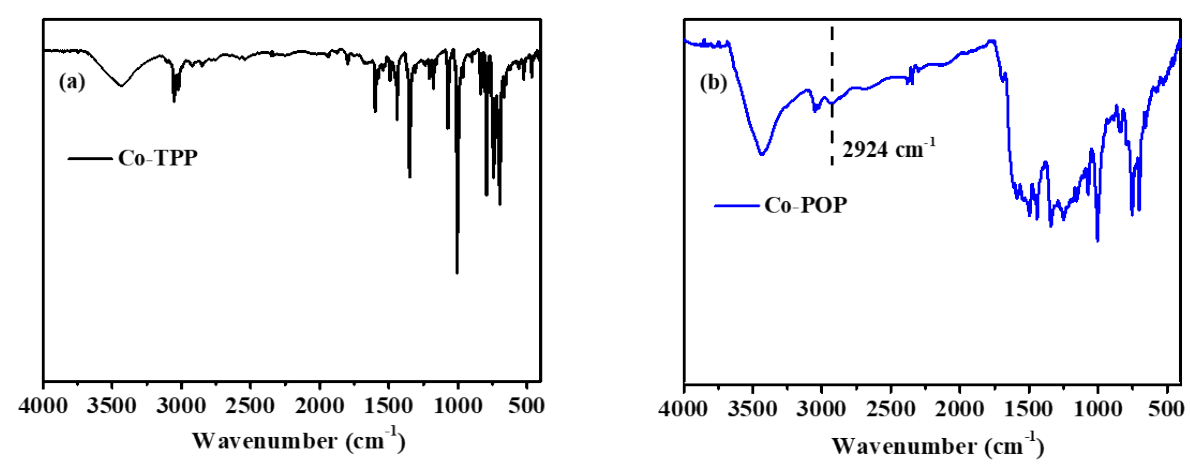

Figure S3. The Infrared absorption spectra of Co-TPP (a) and Co-POP (b). 


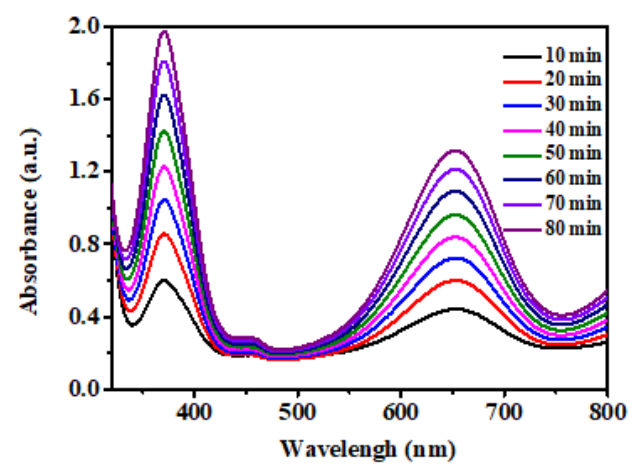

Figure S4. The UV-visible absorption spectra of the Co-POP+TMB system with the extension of time. Reaction conditions: $100 \mu \mathrm{L}$ TMB $(3 \mathrm{mM})$ and $200 \mu \mathrm{L}$ Co-POP solution $(1 \mathrm{mg} / \mathrm{mL})$ were incubated in $2.7 \mathrm{~mL}$ acetate buffer $(\mathrm{pH}=4.0)$ at $25^{\circ} \mathrm{C}$.
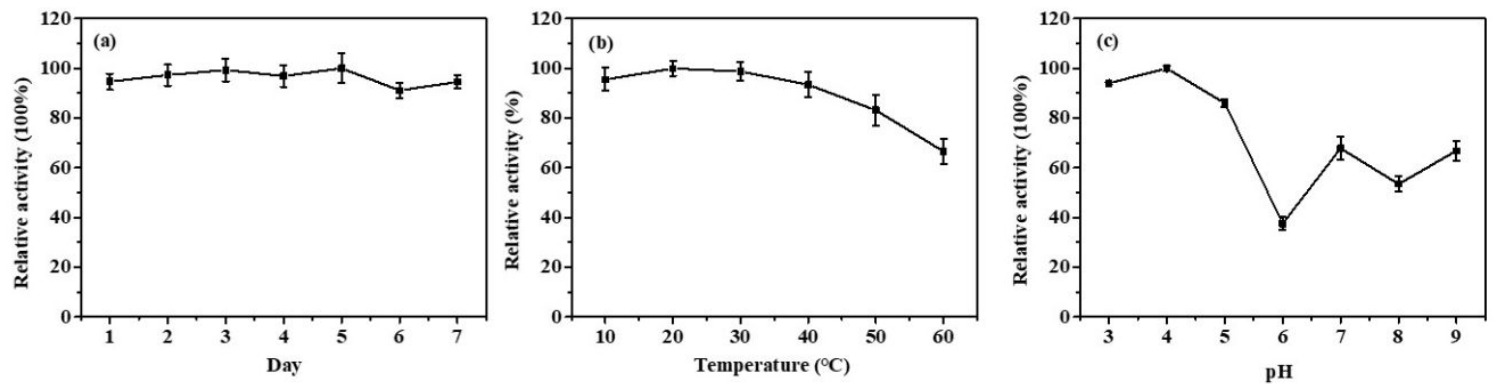

Figure S5. Relative catalytic activity of Co-POP after maintaining at room temperature for 7 days (a); Incubation at different temperatures for $24 \mathrm{~h} \mathrm{(b);} \mathrm{Incubation} \mathrm{at} \mathrm{different} \mathrm{pH}$ values for $24 \mathrm{~h}$ (c). Reaction conditions: $100 \mu \mathrm{L} \mathrm{TMB} \mathrm{(} 3 \mathrm{mM})$ and $200 \mu \mathrm{L}$ Co-POP solution $(1 \mathrm{mg} / \mathrm{mL})$ were incubated in $2.7 \mathrm{~mL}$ acetate buffer $(\mathrm{pH}=4.0)$ at $25^{\circ} \mathrm{C}$ for $30 \mathrm{~min}$. The maximum point in each curve was set as $100 \%$.
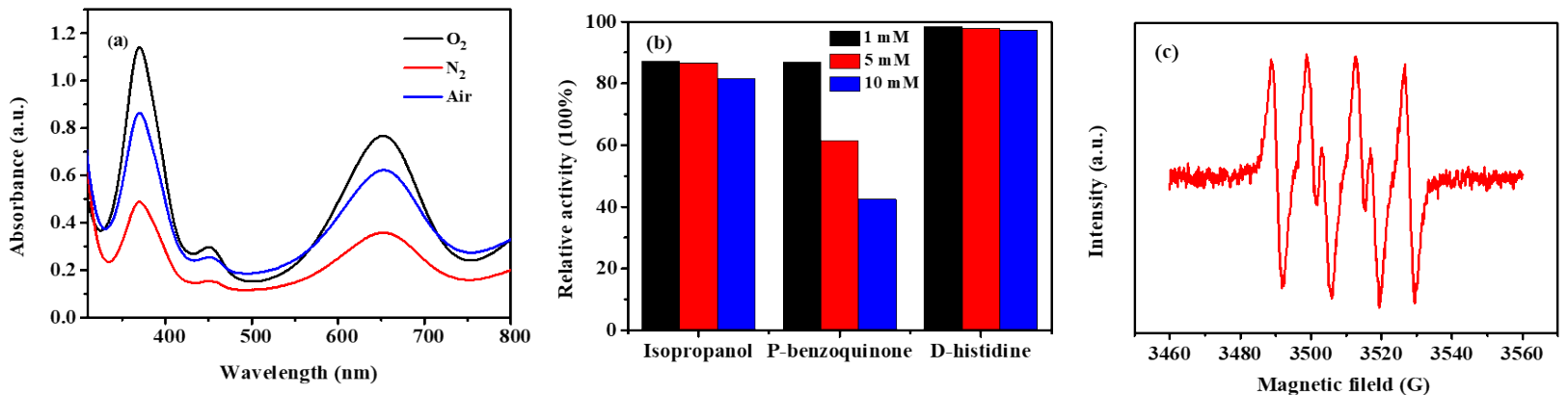

Figure S6. (a) The UV-vis absorption spectra of TMB + Co-POP system in different gas atmospheres for $20 \mathrm{~min}$; (b) Inhibition of different scavenger for TMB+Co-POP system. Reaction conditions: $100 \mu \mathrm{L}$ TMB $(3 \mathrm{mM})$ and $200 \mu \mathrm{L}$ Co-POP solution $(1 \mathrm{mg} / \mathrm{mL})$ were incubated in $2.7 \mathrm{~mL}$ acetate buffer ( $\mathrm{pH}=4.0$ ) at $25^{\circ} \mathrm{C}$ for $30 \mathrm{~min}$; (c) EPR spectra of the Co-POP+DMPO system (DMPO was used as a spin trap). 


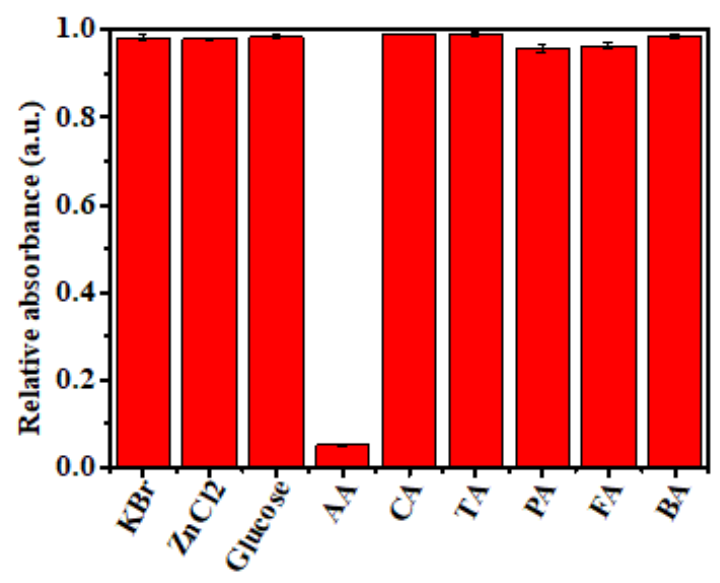

Figure S7. The selectivity analysis of AA detection with different interferents based on CoPOP+TMB system.

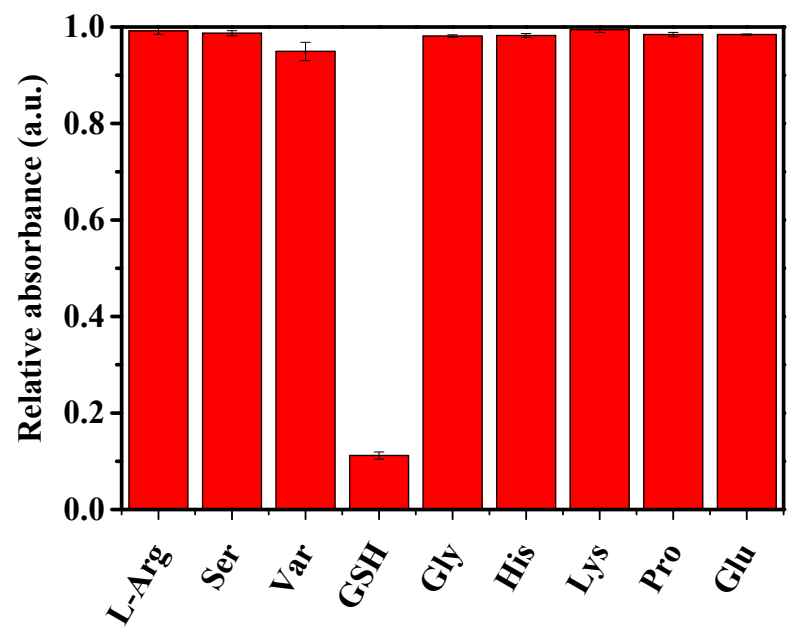

Figure S8. The selectivity analysis of GSH detection with different interferents based on CoPOP+TMB system. 


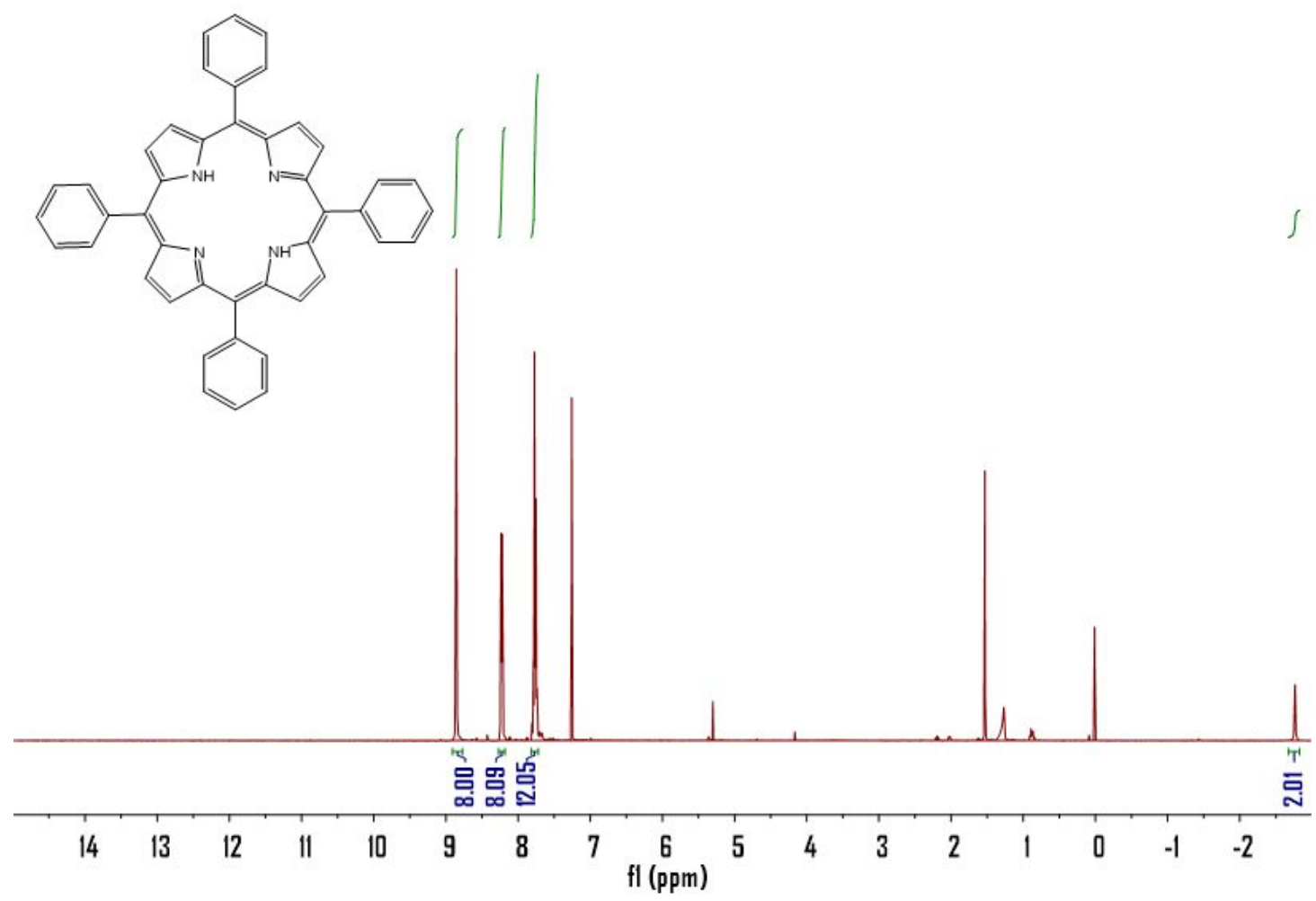

Figure S9. HNMR spectrum of TPP 